\title{
The Efficacy of a Commercial Physical Activity Monitor in Longitudinal Tracking of Pulmonary Hypertension Patients
}

\section{Eliana H Rosenzweig}

Columbia University Vagelos College of Physicians and Surgeons

\section{Gerson Antonio Valencia Villeda}

Arnold Palmer Hospital for Children and Women: Arnold Palmer Hospital for Children

\section{Sarah Crook}

Columbia University Vagelos College of Physicians and Surgeons

\section{Fatima Koli}

Barnard College

\section{Erika B. Rosenzweig ( $\nabla$ esb14@cumc.columbia.edu )}

Columbia University Vagelos College of Physicians and Surgeons https://orcid.org/0000-0003-4849$214 X$

\section{Usha S. Krishnan}

Columbia University Vagelos College of Physicians and Surgeons https://orcid.org/0000-0002-57336096

\section{Research Article}

Keywords: Fitbit, pulmonary hypertension, accelerometry, physical activity, exercise capacity

Posted Date: December 22nd, 2021

DOI: https://doi.org/10.21203/rs.3.rs-1186667/v1

License: (a) (1) This work is licensed under a Creative Commons Attribution 4.0 International License. Read Full License 


\section{The Efficacy of a Commercial Physical Activity Monitor in Longitudinal Tracking of Pulmonary Hypertension Patients}

Eliana Rosenzweig ${ }^{1}$, Gerson Antonio Valencia Villeda ${ }^{1,3}$, Sarah Crook $^{1}$, Fatima Koli ${ }^{2}$, Erika B. Rosenzweig $^{1 *}$, Usha S. Krishnan ${ }^{1 *}$

1. Division of Pediatric Cardiology, Department of Pediatrics Columbia University Irving Medical Center - New York Presbyterian Hospital, New York, NY

2. Data Science Institute, Columbia University, New York, NY

3. The Heart Center at Orlando Health Arnold Palmer Hospital for Children, Orlando, FL *Co-senior Authors

Word Count: 4481

Key words: Fitbit, pulmonary hypertension, accelerometry, physical activity, exercise capacity

Funding Source: Richard M. Bartlett Foundation

\section{Corresponding Author:}

Usha S Krishnan, MD

Professor of Pediatrics

Division of Pediatric Cardiology

Columbia University Medical Center

3959 Broadway CH-2N

New York, NY 10032

PH: 212-305-4436

Fax: 212-342-1443

Email: usk1@cumc.columbia.edu 
Abstract: (word count 245)

Patients with pulmonary arterial hypertension (PAH) have quality of life (QoL) limitations, decreased exercise capacity, and poor prognosis if left untreated. Standard exercise testing is routinely performed for the evaluation of patients with PAH but may be limited in its ability to monitor activity levels in daily living. We evaluated the validity of the commercial Fitbit Charge HR as a tool to assess real time exercise capacity as compared to standard exercise testing in patients with PAH. Ambulatory pediatric and adult PAH patients were enrolled and given a Fitbit with instructions to continuously wear during waking hours. Subjects underwent a 6minute walk test (6MWT), cardiopulmonary exercise test (CPET) and an SF-36 QoL survey on the day of enrollment and follow-up. Twenty-seven ambulatory subjects with PH were enrolled and 21 had sufficient data for analyses(median age 25, range 13-59, $14 \mathrm{~F}$ ) were enrolled. Daily steps measured by the Fitbit had a positive correlation with 6 MWT distance $(r=0.72, p=0.03)$ and an inverse correlation with WHO functional class. On the QoL survey, 77\% reported improvement in energy/fatigue $(p=0.055)$. At follow up there was a strong correlation between Fitbit steps and role limitations due to physical problems $(r=0.88, p=0.020)$ and weaker correlations with less related QoL markers. These findings suggest activity monitors may have potential as a simple/novel method of assessing longitudinal exercise capacity and activity levels in PAH patients. Further study in larger cohorts of patients is warranted to determine the best accelerometric correlates with outcomes. 


\section{Introduction:}

Pulmonary hypertension $(\mathrm{PH})$ is a rare disease characterized by both high mortality and morbidity, often characterized by continuing disease progression despite targeted therapy. Patients with PAH present with multiple symptoms including exertional dyspnea, chest pain, fatigue, syncope, exercise intolerance, and overall poor quality of life. ${ }^{1}$ Often, due to generalized fatigue and fear of worsening $\mathrm{PH}$, patients self-limit activities and become further physically deconditioned. ${ }^{2}$ Historically, there was concern that exercise could worsen right heart function or pulmonary vascular hemodynamics and lead to sudden cardiac related death, leading to patients being discouraged from exercising. However, recent studies have shown that supervised exercise training can be performed by PAH patients without adverse events. ${ }^{3}$ Further, there is increased awareness that exercise training is an important adjunct to pharmacologic therapy and is an integral component of clinical management of PAH. ${ }^{4}$ Exercise-training programs have demonstrated improvements in peak oxygen consumption $\left(\mathrm{VO}_{2}\right)$, WHO Functional Class, hemodynamics, and six-minute walk distance across $\mathrm{PH}$ patients of various etiologies and functional classes..$^{5,6,7}$

The six-minute walk test (6MWT) is the most commonly used serial assessment of exercise capacity in patients with PAH. The 6MWT has a strong independent association with mortality and is a submaximal exercise test initially devised for heart failure patients which correlates well with more extensive cardiopulmonary exercise testing. ${ }^{8}$ The $6 \mathrm{MWT}$ has been validated as a tool to measure functional exercise capacity in patients with chronic respiratory disease ${ }^{9}$ Both the 6MWT and CPET are important tools for evaluation, management and prognostication in patients with PAH. ${ }^{5}$ 
With the expansion of pharmacologic armamentarium in the recent years and increase in innovative transcatheter and surgical interventions that can prolong the lifespan of patients with $\mathrm{PH}$, it is imperative to accurately predict prognosis in $\mathrm{PH}$ patients. Even though these clinical assessments have been well validated, the 6MWT and CPET have some drawbacks. These exercise tests are typically administered once every few months, give an assessment at the timepoint of evaluation, and can only be conducted in hospital or expert clinical settings with trained staff. Thus, they may not accurately represent the activities of daily living in real time for patients, may miss a clinical decline in between assessments, and may not be broadly available for many patients. Furthermore, the 6MWT is less likely to detect clinically significant changes in patients with a WHO FC II or less, due to the tendency to exhibit less severe symptoms. ${ }^{10}$ Additional limitations of the 6MWT may include the individual's motivation, body habitus, patient cooperation, shoe choices and daily conditions. ${ }^{11}$ A multitude of less tangible factors such as comorbid illnesses or anxiety can also alter the results of the patient's distance walked during the 6MWT and the ability to reliably perform exercise during the CPET. ${ }^{12}$

The quantitation of daily activity and sedentary time using accelerometry is a relatively novel endpoint for PAH management. ${ }^{13}$ Accelerometers are well-validated, easy-to-use instruments that quantitate patterns of daily physical activity. ${ }^{14}$ With the modern development of wearable technology, many activity trackers are commercially available and are commonly used in day-today life. Physical activity monitors can track steps per day, distance walked per day, calories burned, physical activity levels throughout the day, and other measures. They have the potential to incentivize patients to advocate for their personalized care and may allow health care providers to gain real-world assessments of patients' daily activity patterns. ${ }^{15}$ The SenseWear armband, a multi-parameter activity tracker used in clinical settings, has been found to correlate 
with the 6MWT data in individual patients as well as most quality-of-life scores. ${ }^{16}$ The Fitbit activity tracker is a commercially available monitor that has been validated in multiple studies and is one of the most widely used monitors in wearable technology. ${ }^{14}$

The primary objective of this study was to determine if there is a relationship between the physical activity data acquired from the Fitbit Charge HR and the results of standard exercise tests and quality of life surveys in patients with $\mathrm{PH}$ in order to gain more insight into the feasibility of using the activity monitor as an assessment of real-world exercise capacity.

\section{Materials and Methods:}

\section{Study population and design:}

Patients $\geq 13$ years of age and ambulatory attending the Pulmonary Hypertension Comprehensive Care Center at the Columbia University Irving Medical Center-New York Presbyterian Hospital with a confirmed diagnosis of PAH and a WHO functional class of I, II, or III symptoms at the time of enrollment were deemed eligible for the study. WHO functional class IV patients were excluded due to the inability to perform serial exercise testing reliably. Potential participants were excluded from the study if they were unable to provide informed consent/assent (as applicable based on age). Patients were also excluded from the study if they had reported usage of a Fitbit or other wearable physical activity trackers in the last six months prior to enrollment. The study was approved by the CUIMC Institutional Review Board (IRB \#AAAQ9188). Informed consent and assent (in children 13-18 years of age) were obtained at the time of enrollment. 
Enrolled participants were given a Fitbit with instructions to wear the device during waking hours with the option to wear the device during sleeping hours. The Fitbit was set up at the PH Center prior to the initial visit and tailored to the patient during the first visit. The name and email were de-identified, and each participant received a number and password assigned to each Fitbit randomly and asked to log into their account weekly to upload data automatically to a secure cloud-based platform created for the purpose of this study. Participants were not given instructions regarding how much exercise or daily physical activity to complete as they were expected to complete in order to obtain the most accurate information about unencouraged, realworld baseline activity levels. Patients were not enrolled at the time of new PH medication initiation.

\section{Baseline Assessment:}

During the initial visit, the participants underwent a 6MWT and CPET. Data from right-heart catheterization and echo assessments performed on or within six months of enrollment were included. All visits were performed as part of standard of care. Patients did not alter medication regimen. Participants also filled out the Short Form 36 survey (SF-36) (Supplement 1), which measures self-reported physical and mental health quality of life in the following eight scales: physical functioning, role limitation due to emotional problems, emotional well-being, general health, change observed in health, pain, social functioning, vitality (energy/fatigue), and role limitation due to physical problems.

\section{Follow-up Assessments:}


Subjects had their post study enrollment follow-up visit after 3-4 months as part of their routine PH clinic follow-up visit. During the follow-up visit, participants underwent standard testing including the 6MWT and CPET as well as a transthoracic echocardiogram (if indicated as part of standard care). Additionally, participants filled out another QoL questionnaire (SF-36) at the first follow up after enrollment. This study focused on the data collected at baseline (specifically the first two weeks of wearing the Fitbit) and the first follow-up visit.

\section{Accelerometry:}

The primary measurement collected was the number of steps per day recorded by the Fitbit activity monitor. The Fitbit classifies activity levels according to respective metabolic equivalents, which calculates exercise intensity taking into account body mass, heart rate, and physical activity to measure the rate of energy expended during periods of physical activity compared to the energy expended during rest. The Fitbit measures sedentary activity as 1.0-1.5 METs, light activity as 1.6-3.0 METs, fair activity as 3.1-6.0 METs, and vigorous activity as greater than 6.0 METs. ${ }^{17,18}$ According to Ainsworth et al., sedentary time typically includes activities such as sitting quietly, sleeping, and watching television. ${ }^{19}$ Lightly active time includes cooking, bathing, and walking $2.0 \mathrm{mi} / \mathrm{h}$ on a level surface. Fairly active activities can include walking a dog, food shopping, and caring for a child. Vigorous activities include swimming, jogging/running, and carrying groceries upstairs.

\section{Statistical methods:}

To assess the association between daily physical activity and clinically relevant endpoints, Spearman correlation was used. Scatterplots of individual patient steps and key variables were 
created to further assess the relationship. To evaluate changes between baseline and follow-up, Wilcoxon signed-rank tests were used to compare differences in steps and other clinically relevant endpoints. The number of steps at baseline and follow-up was calculated as the median number of steps of the 14 days following enrollment and the follow-up visit, respectively. Four days was determined to be the minimum amount of time required to get a valid average of the true activity of the participants. Patients with at least four days of steps in the first two weeks of enrollment were included in this data analysis. Days with $<100$ steps per day were not included.

\section{Results:}

\section{Baseline Demographics:}

The baseline demographics of participants are shown in Table 1.27 participants with PAH were originally enrolled in the study and 21 with sufficient baseline and follow up data were included in the final analysis. Of the six participants who were excluded, one withdrew from the study due to a rash on the wrist area, two did not wear the Fitbit for the first two weeks, and three did not have enough coherent Fitbit data to produce conclusive results. Since most variables are not normally distributed, the median was used to observe the demographics of the participants.

As shown in Table 1, 14 females (66.7\%) and 7 males (33.3\%) were included in the study. 56\% of participants had associated pulmonary arterial hypertension (APAH) and $44 \%$ of the participants had idiopathic pulmonary arterial hypertension (IPAH). The median age of the participants was 25 years (13-59). At baseline 5 (23.8\%) were WHO FC I, 9 (42.9\%) were WHO FC II, and 7 (33.3\%) were WHO FC III. For the baseline period of activity tracking, the Fitbit was worn for a median of 14 days (11.5-14 days). 
Table 2 shows the median daily steps, exercise testing data and SF-36 at baseline and follow-up. The Max RER improved from 1.09 to $1.13, \mathrm{VeVCO}_{2}$ improved, and daily steps measured by the Fitbit improved from 4877.0 to 5324.0 . Additionally, median steps increased by $1,223.5$ steps on average from baseline to follow-up.

\section{Activity Levels:}

Activity levels measured by the Fitbit were compiled from the first two weeks at baseline to determine how the amount of time each day was spent in each respective activity level. The median daily activity level of each participant was calculated, and the average number of minutes spent in each activity level classification was recorded. According to Figure 1, on average, patients spent approximately $82 \%$, or 920 minutes of the day in sedentary level, and 186 minutes, 11 minutes, and 8 minutes in light, fair, and vigorous daily activity levels, respectively, which equates to $16 \%$ of the day spent performing light activity and approximately $1 \%$ of the day performing fair and vigorous activity.

There was a trend toward fewer daily steps as the WHO Functional class increased in severity (Figure 2). When the median daily steps were compared to the WHO FC of each participant, Class I had the broadest range of median daily steps. The average daily steps taken by WHO FC 1 was 6,440 and for FC II and III were 6,121 and 4,902 steps, respectively. From WHO FC I to II the mean steps taken each day decreased approximately by 319 steps per day and from functional class II to III the mean steps taken decreased by 1,219 steps per day. The mean line 
also supports an overall decrease trend in steps with increasing PH severity, with greater decrease from functional class II to functional class III.

\section{Quality of Life:}

The results of the SF-36 questionnaire at baseline and at 3-4 month follow up were compared in 13 subjects who completed their follow-up surveys. Self-reported physical functioning, role limitation due to emotional problems, emotional well-being, general health, and change observed in health were not significantly improved. However, as seen in Table 2, perceived pain, social functioning, vitality (energy/fatigue), and role limitation due to physical problems improved but due to limited numbers, the change did not reach statistical signficance. Vitality scores improved the most in the non-restricted sample from baseline to 3-month follow-up $(p=0.055)$ with $77 \%$ of patients reporting improvement in their energy and fatigue scores. The average energy and fatigue score was 57.6 and improved to 62 at follow-up. Self-reported physical function scores improved in $38 \%$ of participants with an average baseline physical function score of 70.0 and an increased score of $80.0 .77 \%$ of subjects reported either an improvement in role limitations due to emotional problems or no change from a baseline score of 50 to $75.54 \%$ of participants reported no change or an improvement in role limitations due to physical health. Approximately $70 \%$ of the participants reported an improvement or no change in their social functional quality of life score with the average score improving from 75 to 100 at follow up. Participants perceived an improved average overall health change scores of 50 to 75.0 on follow-up.

\section{Exercise:}


The Spearman correlation was used as a pairwise analysis to determine if steps measured by the Fitbit correlate with exercise parameters measured by CPET/6MWD tests or the SF-36 QoL questionnaire.The correlation coefficients between steps and other clinical endpoints are presented in Table 3. The 6MWD in meters was found to have a strong positive correlation with steps measured by the Fitbit Charge HR. The Spearman's rank correlation coefficient was determined to be 0.72 at follow-up $(p=0.03)$. A trend can be observed between the meters walked during the 6MWT and steps measured by the Fitbit (Figure 3, 4). Participants who are able to walk more meters during the 6MWT frequently walk more steps per day as measured by the Fitbit monitor. There are fewer participants graphed at follow-up; however, the correlation between 6MWD and steps measured by the Fitbit is noticeably stronger at follow-up (Figure 4). In fact, a majority of other clinical endpoints had a stronger correlation at follow-up as compared to baseline, including Borg index, $\mathrm{VO}_{2}$, Watts, and $\mathrm{O}_{2}$ saturation as well as all SF-36 classifications (Table 3).

The SF-36 survey found a strong positive correlation between steps and vitality at followup with a Rho correlation of 0.71 . This correlation is demonstrated in Figure 5 by the positive linear trend of Fitbit steps plotted against vitality at follow-up. In addition, there was a significantly strong correlation of 0.88 between baseline to follow-up for the role limitation due to physical problems $(\mathrm{p}=0.020)$. Physical functioning and general health showed moderate correlation to steps walked, with correlation coefficients of $0.41,0.51$ and 0.52 , respectively. The Spearman correlation was weaker between steps and health change, pain, and role limitations due to emotional problems, with correlations of $0.22,0.27$, and 0.37 , respectively. Social functioning and emotional well-being showed moderate correlations with 0.52 and 0.56 , respectively. 


\section{Discussion:}

Patients with PH have significant compromise in activities of daily living. Use of New York Heart Association (NYHA) or World Health Organization (WHO) functional classification can be inadequate as patients with chronic illness often do not have great insights into their exercise capacity due to life-long self limitation of activity. ${ }^{15}$ Thus, exercise testing measures such as 6MWD and CPET have been very valuable tools to measure their exercise capacity at baseline and in response to therapy. However, these tests measure exercise capacity at a given point in time, whereas use of activity monitors measure exercise capacity for a period of time including during activities of daily living. A wearable accelerometer, even a commercial physical activity tracker such as the Fitbit, can provide fairly valid and measurable real time assessment of a patient's effort, tolerance, and activity levels and can prove a valuable tool for clinical management as well as a measurable validated endpoint for therapeutic research. A recent study published by Zijlstra et al. found that accelerometry may provide clinically meaningful endpoints in children with $\mathrm{PAH} .{ }^{20}$ This may be of value while evaluating pediatric patient's response to therapy and interventions.

Despite PH patients having markedly decreased exercise capacity and compromised respiratory function, a strong correlation was observed between the 6MWD and real life exercise variables in patients over 13 years of age, including steps measured by the activity monitor as well as selfreported health-related quality of life, most noticeably vitality. The SF-36 survey showed a stronger correlation to variables of role limitation due to physical problems than emotional problems from baseline to follow-up and a strong correlation with self-reported vitality at 
follow-up. The predictibility of the activity monitor for exercise parameters was supported by strong correlations with exercise variables such as $6 \mathrm{MWD}$ and $\mathrm{O}_{2}$ saturation during exercise. The correlations between 6MWD and exercise/SF-36 survey variables strengthened at follow-up as compared to baseline, which suggests the usability of the Fitbit activity tracker as a longitudinal measure of exercise capacity with improved correlation suggesting more consistent use and possibly, an incentive to be more active while wearing a measurable activity tracker.

As expected, the average 6MWD as well as measured steps by the Fitbit were lower than the normal reference point for healthy subjects and were further decreased with each level of WHO FC..$^{21,22}$ The 6MWD (in meters) showed a strong positive correlation to Fitbit measured steps, indicating that Fitbit steps can be a potentially valid real-time tracking tool. Participants who can walk more meters on a 6MWT will typically walk more steps on a Fitbit. Additionally, participants with more follow up data were shown to have a stronger correlation between the steps measured by the Fitbit and the 6MWD as well. As the Fitbit activity tracker accrues more data, the data can potentially be more reflective of the disease state in conjunction with exercise and QoL parameters. This supports the potential usability of activity tracking as a longitudinal measure of real-time daily tracking for PH patients as opposed to short-term standard exercise tests, which hold limitations in a clinical setting and are also considered to be indirect outcome measures in the context of clinical trails for PAH patients. ${ }^{10,12,23}$ Sehgal, et al. reported similar results using the Fitbit Charge HR, corroborating the correlation of steps to 6MWT endpoints, ultimately favoring a long-term wrist-worn accelerometer in PAH patients. ${ }^{22}$ 
Prior studies have found a correlation between sedentary activity levels and QoL whereas the current study found a correlation between some QoL physical variables to steps on the activity tracker, suggesting further exploration into the exact relationship between these variables. ${ }^{9}$ Some endpoints measured by the SF-36 quality of life questionnaire were strongly correlated to the steps measured by the Fitbit at follow-up, including the measurement of vitality. The vitality score is comparable to the Borg dyspnea score on the $6 \mathrm{MWT}$, with vitality as a longer term variable or endpoint of percieved respiratory function. Lima et al. highlighted poor quality of life as a significant factor impacting patients with PAH. ${ }^{24}$ The SF-36 questionnaire was observed to have a lower overall correlation in less related areas including role limitations due to emotional problems and pain, and was more strongly correlated with physical activity outcomes, including role limitations due to physical pain from baseline to follow-up and vitality reported at followup. This suggests the Fitbit tracker as a potential clinical tool to track perceived fatigue, during or after physical activity, which is a commonly reported symptom of $\mathrm{PH}$.

Activity monitor data correlated more strongly to physical activity parameters like 6MWD rather than those dependent on ventilatory function such as Ve/VCO2. Similar to the current study, these variables representative of ventilatory function or other physiological variables have not been studied extensively or found to be signficant in previous studies. ${ }^{13,16}$ In addition, the significantly strong correlation between activity tracked steps and role limitations due to physical activity from baseline to follow-up suggests that the Fitbit may may be a valid predictor of exercise capacity over time and physical activity outcomes in PH patients. In addition, correlation measurements with 6MWD were stronger at follow-up suggesting the exploration of long-term accelerometry in PH patients may be more beneficial than a short-term use. A study 
done by Okumus et al. observed an association between 6MWD and physical activity as well as QoL using a SneseWear arm band activity tracker. ${ }^{16}$ The average steps taken daily measured by the Fitbit decreased with increased WHO FC, further suggesting usage of daily steps as a potential marker of disease severity in $\mathrm{PH}$ patients, as a more accurate way to measure functional class in patients, and to help risk stratification and prognostication (Figure 2).

Participants were not given any instructions regarding expectations of physical activity output in order to determine if the Fitbit activity tracker alone motivates patients will pulmonary hypertension to exercise. Although most changes in exercise testing outcomes were insignificant, when exercise tests were analyzed at baseline vs. follow-up, it was observed that some participants individually did improve their scores from baseline to follow-up. Surprisingly the 6MWD decreased from baseline to follow-up, while the daily steps measured by the Fitbit increased during this period, though neither were statistically significant (Table 2). Patients did not change medication regimen through the study. In only 3-4 months, which was the main area of analysis to ensure reliability of accelerometry results, a significant improvement in exercise parameters was not altogether expected but a lack of decline is in itself of clinical importance. Self-reported endpoints of the SF-36 survey including vitality and role limitation due to physical activity did improve, suggesting that participants may percieve an improvement before one can be detected by standard exercise testing. In previous studies, exercise training has been seen to significantly improve quality of life, $6 \mathrm{MWD}, \mathrm{WHO} \mathrm{FC}, \mathrm{VO}_{2}$, systolic pulmonary artery pressure, and overall exercise capacity in patients with pulmonary hypertension. ${ }^{4,6,21}$ In order to clarify whether the Fitbit can stand-alone as a motivator for patients to exercise, there is a need for clinical intervention, such as an exercise program that could be paired with Fitbit or other 
activity tracking technology as a comparison. Thus, it is too early to say whether the Fitbit alone can be a motivational factor of clinical improvement in physical activity and disease outcomes.

\section{Limitations:}

This study is limited by the relatively small sample size and future larger multicenter studies are required to further investigate the utility of activity monitors in pulmonary hypertension. Certain trends, specifically, measurements at baseline and follow-up, may not have been statistically significant due to a small sample size. Because the study sought to look at a commercially available physical activity monitor, the study may also be limited by the variables provided by the Fitbit, as opposed to standardized measurements provided by other accelerometers built for research purposes. Moreover, the participants analyzed were somewhat heterogeneous with both children and adults included so there may be differences in activity levels that should be explored separately in these groups to determine any significant differences.

\section{Conclusion:}

In summary, the correlation between Fitbit measured steps and 6MWD suggests that the Fitbit has potential benefit to act as an effective way to monitor exercise capacity in patients with $\mathrm{PH}$ or even as an adjunct to short-term 6MWT and CPET exercise tests and could be an excellent exploratory endpoint for future studies. Future studies utilizing a larger sample size, or an exercise intervention are warranted to determine whether daily activity monitoring has an important role in the assessment and management of patients with pulmonary hypertension and other cardiovascular diseases. 


\section{Acknowledgements:}

The authors acknowledge the contribution of Anuj Katiyal, MA for assistance in data platform construction. The authors would like to acknowledge the Richard M. Bartlett Foundation for their generous support of this research.

Funding: This research was made possible in part by support from The Richard M. Bartlett Foundation's research grant funding at The Pulmonary Hypertension Comprehensive Care Center at Columbia University Irving Medical Center.

\section{References:}

1. Galie N, Manes A, Negro L, et al. A meta-analysis of randomized controlled trials in pulmonary arterial hypertension. Eur Heart J 2009; 30: 394-403

2. Mainguy V, Provencher S, Maltais F, et al. Assessment of Daily Life Physical Activities in Pulmonary Arterial Hypertension. PLoS One 2011; 6:e27993.

3. Fowler RM, Gain KR, and Gabbay E. Exercise Intolerance in Pulmonary Arterial Hypertension. Pulm Med 2012; 2012: 359204.

4. Mereles D, Ehlken N, Grunig E, et al. Exercise and respiratory training improve exercise capacity and quality of life in patients with severe chronic pulmonary hypertension. Circulation 2006; 114:1482-1489.

5. Badesch BD, Champion HC, Torbicki A, et al. Diagnosis and assessment of pulmonary arterial hypertension. J Am Coll Cardiol 2009; 54:55-56.

6. Grunig E, Lichtblau M, Nagel C, et al. Safety and efficacy of exercise training in various forms of pulmonary hypertension. Eur Respir J 2012; 40:84-92. 
7. Nagel C, Prange F, Grunig E, et al. Exercise training improves exercise capacity and quality of life in patients with inoperable or residual chronic thromboembolic pulmonary hypertension. PLoS One 2012; 7:e41603.

8. Sehgal, S., Chowdhury A, Highland, K. B., et al. Counting steps: A new way to monitor patients with pulmonary arterial hypertension. Lung 2019; 197:501-508.

9. Frost AE, Langleben D, Oudiz R, et al. The 6-min walk test (6MWT) as an efficacy endpoint in pulmonary arterial hypertension clinical trials: demonstration of a ceiling effect. Vascul Pharmacol 2005; 43:36-39.

10. American Thoracic Society ATS statement: guidelines for the six-minute walk test. Am $J$ Respir Crit Care Med 2002; 166: 111-117.

11. Reybrouck T. Clinical usefulness and limitations of the 6-minute walk test in patients with cardiovascular or pulmonary disease. Chest. 2003; 123:325-327.

12. Pugh ME, Buchowski MS, Hemnes AR, et al. Physical activity limitation as measured by accelerometry in pulmonary arterial hypertension. Chest. 2012; 142:1391-1398.

13. Evenson KR, Goto MM, Furberg RD. Systematic review of the validity and reliability of consumer-wearable activity trackers. Int J Behav Nutr Phys Act 2015; 12:159.

14. E. Chiauzzi, C. Rodarte and P. DasMahapatra, Patient-centered activity monitoring in the self-management of chronic health conditions. BMC Med 2015; 13:77.

15. Okumus G, Aslan G K, and Kiyan E, et al. The role of an activity monitor in the objective evaluation of patients with pulmonary hypertension. Clin Respir J 2018; 12:119-125.

16. Godfrey A, Conway R, O’Laighin G. Direct measurement of human movement by accelerometry. Med Eng Phys. 2008; 30(10):1364-1386. 
17. Jetté M, Sidney K, Blümchen G. Metabolic equivalents (Mets) in exercise testing, exercise prescription, and evaluation of functional capacity. Clin Cardiol 1990;13(8):555-565.

18. Ainsworth BE, Haskell WL, Leon AS, et al. Compendium of physical activities: classification of energy costs of human physical activities. Med Sci Sports Exerc 1993; 25(1):71-80.

19. Zijlstra WH, Poegstra MJ. Berger RM, et al. Physical activity in pediatric pulmonary arterial hypertension measured by accelerometry. A candidate clinical endpoint. American Journal of Respir Crit Care Med 2017; 196(2): 220-227.

20. Demir R, Kucukoglu M. Evaluation of exercise capacity in pulmonary hypertension. Arch Turk Soc Cardiol 2010; 38:580-588.

21. C. Casanova. The 6-min walk distance in healthy subjects: reference standards from seven countries. European Respiratory Journal 2011; 37:150-156.

22. Cowdhury A, Sehgal S, Highland K, et al. Accuracy of Accelerometry: Measured Distance and Heart Rate in Patients with Pulmonary Arterial Hypertension. American Journal of Respir Crit Care Med 2017; 195:A3116.

23. Lima, L, Mendes F, Pereira M, et al. Factors associated with daily physical activity in patients with pulmonary arterial hypertension. Eur Respir J 2019; 4: Suppl. 63, PA5465. 


\section{Declarations section:}

\section{Funding}

This research was made possible in part by support from The Richard M. Bartlett Foundation's research grant funding at The Pulmonary Hypertension Comprehensive Care Center at Columbia University Irving Medical Center.

The authors declare that no funds, grants, or other support were received during the preparation of this manuscript

\section{Competing Interests}

Financial interests: Erika Berman Rosenzweig and Usha Krishnan's institution have received research grant support from Actelion-Janssen, Bayer, SonVie, and United Therapeutics. There are no additional relevant financial or non-financial interests to disclose.

\section{Author Contributions}

All authors contributed to the study conception and design. Material preparation, data collection and analysis were performed by Eliana Rosenzweig, Sarah Crook, Fatima Koli. The first draft of the manuscript was written by Eliana Rosenzweig and Erika Rosenzweig and Usha Krishnan, and all authors commented on previous versions of the manuscript. All authors read and approved the final manuscript.

\section{Ethics approval}

This study was performed in line with the principles of the Declaration of Helsinki. The study was approved by the CUIMC Institutional Review Board (IRB \#AAAQ9188). Informed consent and assent (in children 13-18 years of age) were obtained at the time of enrollment.

\section{Consent to participate}

Informed consent/assent was obtained from all individual participants included in the study. 
Table 1: Baseline Demographics \begin{tabular}{|l|l|}
\hline $\mathbf{N}=21$ & Median (IQR) / n (\%) \\
\hline
\end{tabular} 


\begin{tabular}{|l|l|}
\hline Age (years) & $25.0(13$ to 59$)$ \\
\hline Sex (female) & $14(66.7)$ \\
\hline Height (cm) & $162.0(157.0$ to 168.0$)$ \\
\hline Weight (kg) & $57.7(54.0$ to 74.0$)$ \\
\hline BMI & $21.8(20.5$ to 28.0$)$ \\
\hline BSA & $1.65(1.56$ to 1.87$)$ \\
\hline WHO FC & \\
\hline I & $5(23.8)$ \\
\hline II & $9(42.9)$ \\
\hline III & $7(33.3)$ \\
\hline
\end{tabular}

Baseline demographics were compiled using the median and interquartile range. BMI: Body Mass Index; BSA: Body Surface Area; WHO FC: World Health Organization Functional Class (I-III)

\section{Table 2a: Exercise and SF-36 Variables Baseline vs. Follow-up}

\begin{tabular}{|c|c|c|c|c|}
\hline & Baseline $(\mathrm{N}=\mathbf{2 1})$ & Follow-up $(\mathrm{N}=16)$ & Change & P-value \\
\hline \multicolumn{5}{|l|}{ Fitbit } \\
\hline Steps ( $\geq 4$ days) & $4877.0(3418.5$ to 7327.0$)$ & $5324.0(4804.5$ to 8422.5$)$ & $1022.5(-138.5$ to 2200.0$)$ & 0.084 \\
\hline Steps ( $\geq 7$ days) & $5081.0(3288.3$ to 7898.3$)$ & $5534.0(4902.0$ to 8422.5$)$ & $1022.5(807.0$ to 2189.0$)$ & \\
\hline \multicolumn{5}{|l|}{ Exercise testing } \\
\hline 6MWD (m) & 480.5 (434.0 to 526.0 ) & $473.0(427.5$ to 569.5$)$ & $-21.0(-68.0$ to 13.5$)$ & 0.188 \\
\hline 6MWT (steps) & $635.0(584.0$ to 722.5$)$ & - & - & \\
\hline Borg & $2.0(0.5$ to 3.0$)$ & $2.0(0.5$ to 3.0$)$ & $0(-0.5$ to 0.0$)$ & \\
\hline $\mathrm{VO}_{2} \max (\mathrm{ml} / \mathrm{kg} / \mathrm{min})$ & $16.5(14.0$ to 21.1$)$ & $18.7(15.0$ to 28.8$)$ & $-0.4(-1.5$ to 0.6$)$ & 0.820 \\
\hline $\mathrm{VO}_{2} \max (\%$ pred.) & $47.0(36.5$ to 57.5$)$ & $53.0(41.0$ to 67.0$)$ & $-1.0(-6.0$ to 2.0$)$ & \\
\hline Watts & $75.0(49.0$ to 96.0$)$ & $51.5(42.0$ to 89.0$)$ & $-3.0(-4.0$ to 1.0$)$ & 0.250 \\
\hline Watts (\% pred.) & $52.5(35.0$ to 60.0$)$ & $35.0(33.0$ to 58.0$)$ & $-2.0(-2.0$ to 1.0$)$ & \\
\hline Max RER & $1.10(1.05$ to 1.20$)$ & $1.13(1.10$ to 1.19$)$ & $0.10(0.01$ to 0.12$)$ & 0.078 \\
\hline $\mathrm{PetCO}_{2}$ & $28.0(23.5$ to 33.5$)$ & $24.5(22.8$ to 31.0$)$ & $-0.8(-1.5$ to 2.5$)$ & \\
\hline $\mathrm{VeVCO}_{2}$ & $40.0(35.0$ to 44.0$)$ & $41.0(35.0$ to 47.0$)$ & $4.5(2.0$ to 7.0$)$ & \\
\hline
\end{tabular}




\begin{tabular}{|c|c|c|c|c|}
\hline $\mathrm{O}_{2}$ sat rest $(\mathrm{CPET})$ & $97.0(96.0$ to 98.0$)$ & $96.5(94.5$ to 98.0$)$ & $0.5(-1.5$ to 1.0$)$ & \\
\hline $\mathrm{O}_{2}$ sat exercise $(\mathrm{CPET})$ & $94.0(92.0$ to 97.0 & 54.0 & -11.0 & \\
\hline $\mathrm{O}_{2}$ sat rest $(6 \mathrm{MWT})$ & $98.0(96.0$ to 98.0$)$ & $98.0(97.0$ to 98.0$)$ & $1.0(0.0$ to 2.0$)$ & \\
\hline \multicolumn{5}{|l|}{ SF-36 } \\
\hline Physical functioning & $70.0(45.0$ to 90.0$)$ & $80.0(45.0$ to 85.0$)$ & & 1.000 \\
\hline Role limitations due to physical problems & $50.0(0.0$ to 100.0$)$ & $75.0(75.0$ to 75.0$)$ & & 0.500 \\
\hline $\begin{array}{l}\text { Role limitations due to emotional } \\
\text { problems }\end{array}$ & $100.0(100.0$ to 100.0$)$ & $83.3(66.7$ to 100.0$)$ & & 0.750 \\
\hline Energy/fatigue & $57.5(50.0$ to 65.0$)$ & $62.5(55.0$ to 65.0$)$ & & 0.246 \\
\hline Emotional well-being & $80.0(72.0$ to 80.0$)$ & $72.0(64.0$ to 88.0$)$ & & 1.000 \\
\hline Social functioning & $75.0(50.0$ to 87.5$)$ & $100.0(62.5$ to 100.0$)$ & & 0.500 \\
\hline Pain & $93.8(57.5$ to 100.0$)$ & $93.8(67.5$ to 100.0$)$ & & 0.500 \\
\hline General health & $35.0(30.0$ to 65.0$)$ & $30.0(25.0$ to 70.0$)$ & & 0.844 \\
\hline Health change & $50.0(50.0$ to 50.0$)$ & $75.0(50.0$ to 75.0$)$ & & 1.000 \\
\hline
\end{tabular}

Data is presented as median with IQR. The overall sample was used for baseline data. Follow-up data as well as the change score used the restricted sample. Change score represents the median of individual change scores. 6MWD: 6-min walk distance; 6MWT: 6-min walk test; Borg: Borg Dyspnea Score; $\mathrm{VO}_{2}$ max: maximal oxygen uptake; Watts: exercise resistance; Max RER: maximum respiratory exchange ratio; PetCO 2 : partial pressure of end tidal $\mathrm{CO}_{2} ; \mathrm{VeVCO}_{2}: \mathrm{VE} / \mathrm{VCO}_{2}$ or ventilation equivalent for carbon dioxide. Refer to Supplementary Table $2 \mathrm{~b}$ for sample sizes (n) of each variable.

Table 3: Spearman Correlation (pairwise) of Exercise and Survey Variables at baseline and follow-up:

\begin{tabular}{|c|c|c|c|c|c|c|}
\hline & \multicolumn{2}{|c|}{ Baseline } & \multicolumn{2}{|c|}{ Follow-up } & \multicolumn{2}{|l|}{ Change } \\
\hline & Rho & $\begin{array}{c}P \\
\text { value }\end{array}$ & Rho & $P$ value & Rho & $P$ value \\
\hline 6MWD (m) & 0.37 & 0.161 & 0.72 & 0.030 & -0.46 & 0.257 \\
\hline 6MWT (steps) & 0.33 & 0.176 & & & & \\
\hline Borg & 0.29 & 0.256 & 0.32 & 0.400 & -0.41 & 0.310 \\
\hline $\mathrm{VO}_{2}(\mathrm{ml} / \mathrm{kg} / \mathrm{min})$ & 0.02 & 0.950 & 0.12 & 0.765 & -0.17 & 0.668 \\
\hline
\end{tabular}




\begin{tabular}{|c|c|c|c|c|c|c|}
\hline $\mathrm{VO}_{2}(\%$ pred. $)$ & -0.18 & 0.452 & 0.33 & 0.381 & -0.17 & 0.668 \\
\hline Watts & 0.23 & 0.384 & 0.37 & 0.469 & -0.35 & 0.499 \\
\hline Watts (\% pred.) & 0.18 & 0.494 & 0.38 & 0.462 & -0.21 & 0.686 \\
\hline Max RER & 0.44 & 0.052 & -0.17 & 0.667 & -0.40 & 0.286 \\
\hline $\mathrm{P}_{\mathrm{ETCO}}{ }^{2}$ & 0.27 & 0.248 & 0.20 & 0.629 & -0.25 & 0.548 \\
\hline $\mathrm{VeVCO}^{2}$ & 0.38 & 0.318 & -0.03 & 0.957 & -1.00 & 1.00 \\
\hline $\mathrm{O}_{2}$ sat rest $(\mathrm{CPET})$ & 0.11 & 0.649 & -0.22 & 0.606 & -0.12 & 0.774 \\
\hline $\mathrm{O}_{2}$ sat exercise (CPET) & -0.14 & 0.787 & -0.80 & 0.200 & & \\
\hline $\mathrm{O}_{2}$ sat rest $(6 \mathrm{MWT})$ & 0.00 & 1.000 & -0.12 & 0.827 & -0.30 & 0.624 \\
\hline $\mathrm{O}_{2}$ sat exercise (6MWT) & 0.37 & 0.469 & -0.40 & 0.505 & & \\
\hline Physical functioning & 0.22 & 0.354 & 0.41 & 0.425 & -0.62 & 0.191 \\
\hline Role limitations due to physical problems & -0.09 & 0.720 & 0.51 & 0.305 & 0.88 & 0.020 \\
\hline Role limitations due to emotional problems & -0.27 & 0.256 & 0.37 & 0.470 & 0.58 & 0.231 \\
\hline Energy/fatigue & -0.04 & 0.886 & 0.71 & 0.117 & 0.03 & 0.957 \\
\hline Emotional well-being & -0.22 & 0.352 & 0.56 & 0.322 & 0.70 & 0.188 \\
\hline Social functioning & 0.05 & 0.828 & 0.52 & 0.295 & 0.20 & 0.747 \\
\hline Pain & -0.04 & 0.854 & 0.27 & 0.600 & 0.29 & 0.577 \\
\hline General health & 0.39 & 0.092 & 0.52 & 0.295 & -0.31 & 0.552 \\
\hline Health change & 0.07 & 0.773 & 0.22 & 0.718 & -0.26 & 0.668 \\
\hline
\end{tabular}

\section{Figure Legends:}


1: Baseline activity levels were compiled from the Fitbit in the first week of monitoring. The activity levels were broken down into sedentary, light, fair, and vigorous activity.

2: The average daily step count of each participant was plotted over the first two weeks of monitoring and graphed against WHO FC I, II, and III.

3: Vitality scores of self-reported energy/fatigue measured by the SF-36 survey were compared in 13 participants at baseline and follow-up.

4a: Six-minute walk distance in meters was graphed in a scatterplot against median steps at baseline (first two weeks of Fitbit monitoring).

4b: Six-minute walk distance in meters was graphed in a scatterplot against median steps at follow-up (two weeks of Fitbit monitoring after 3-4 months).

5: Median Fitbit measured steps at follow-up (two weeks of Fitbit monitoring after 3-4 months) was graphed in a scatterplot against SF-36 self-reported vitality

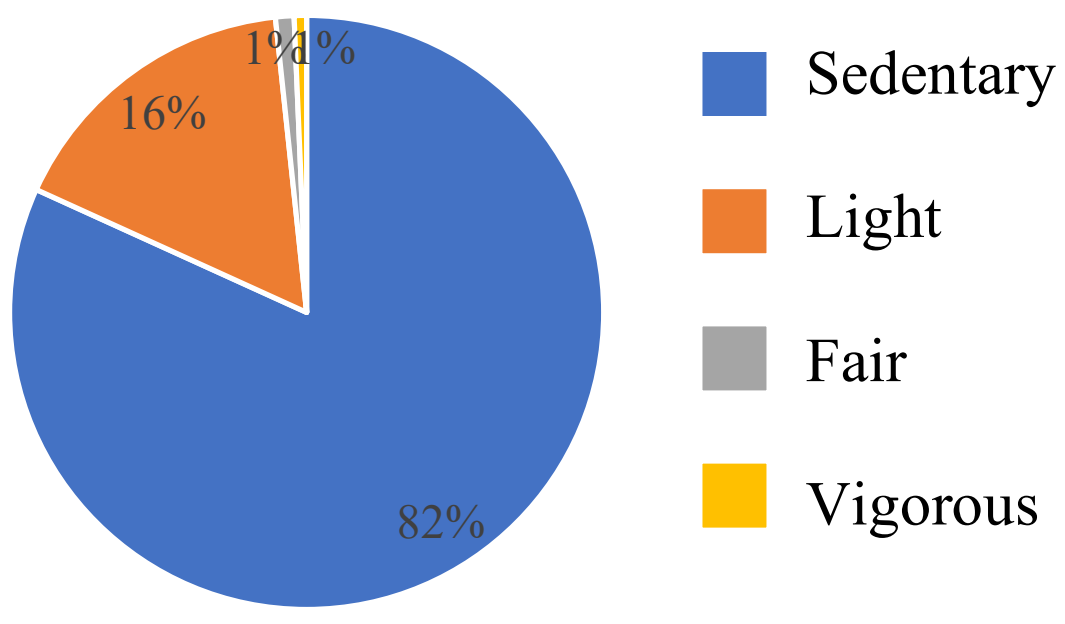

Figure 1: Average Daily Activity Level Spent in Minutes 


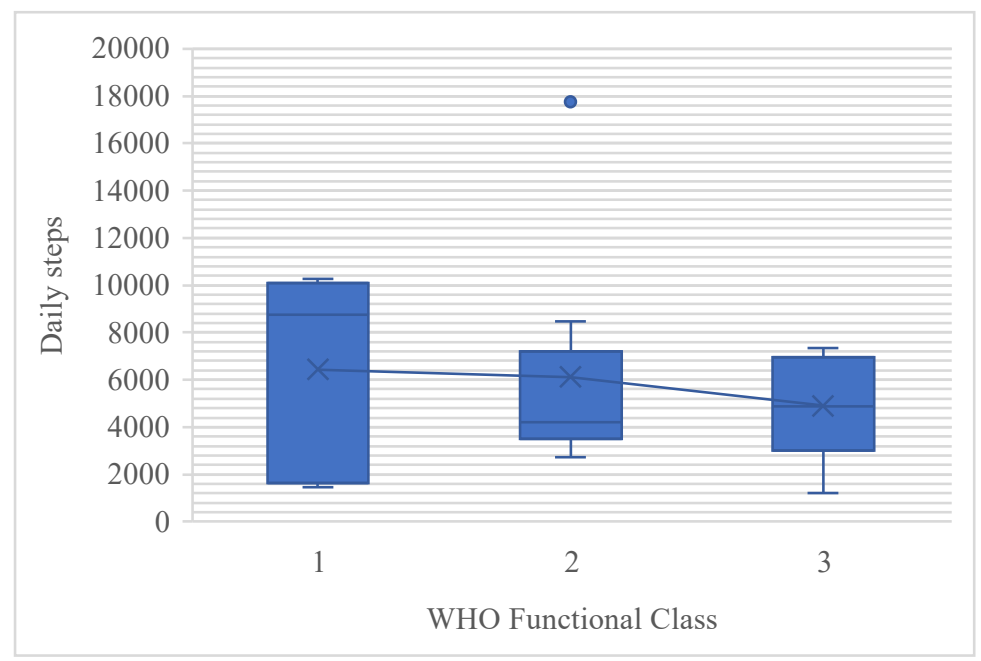

Figure 2: Daily Fitbit Steps and WHO FC

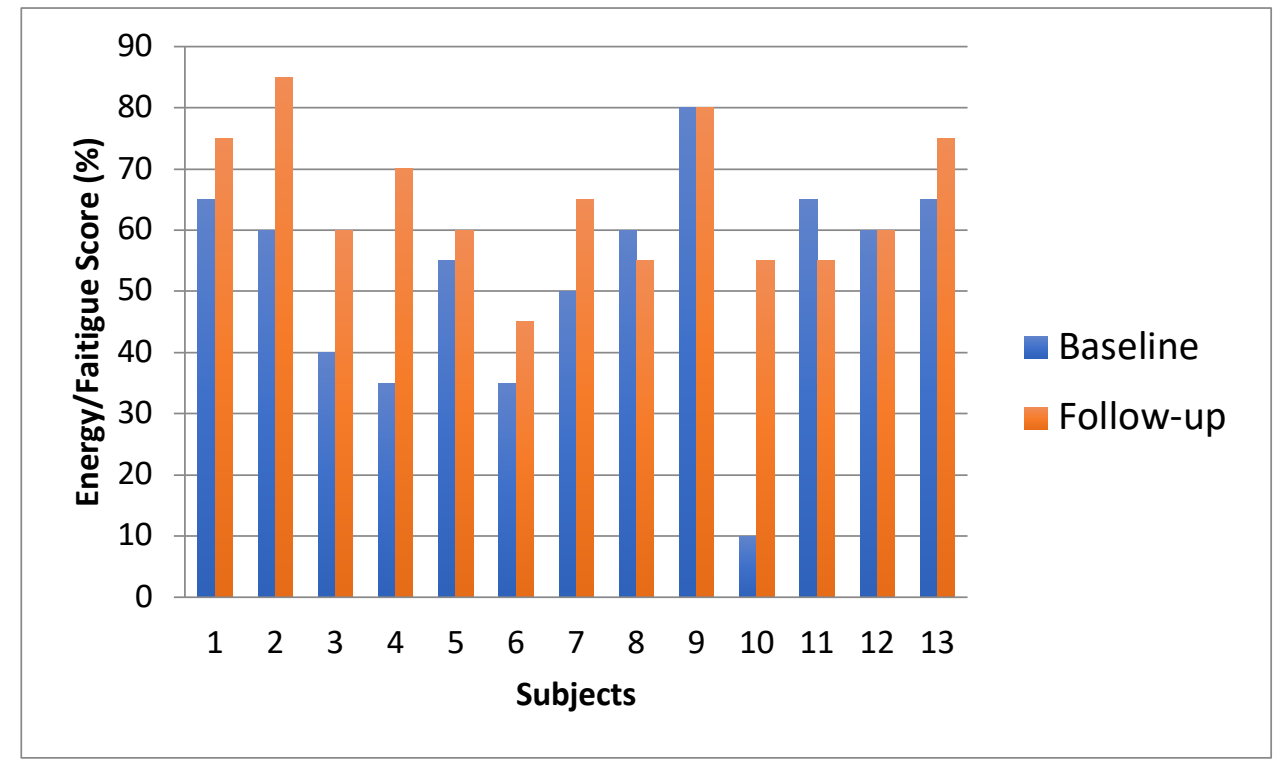

Figure 3: SF-36 QoL Vitality Scores (baseline vs. follow-up, n=13) 


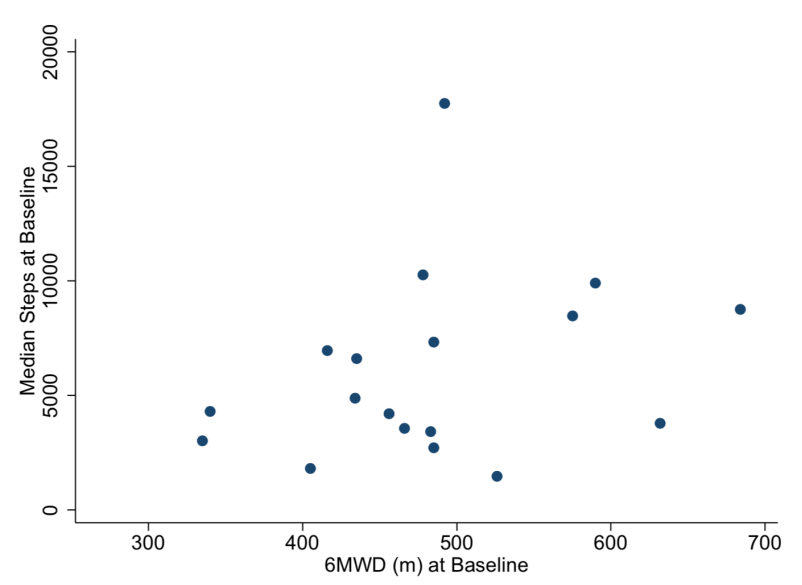

Figure 4a: 6MWD vs Fitbit Steps at Baseline

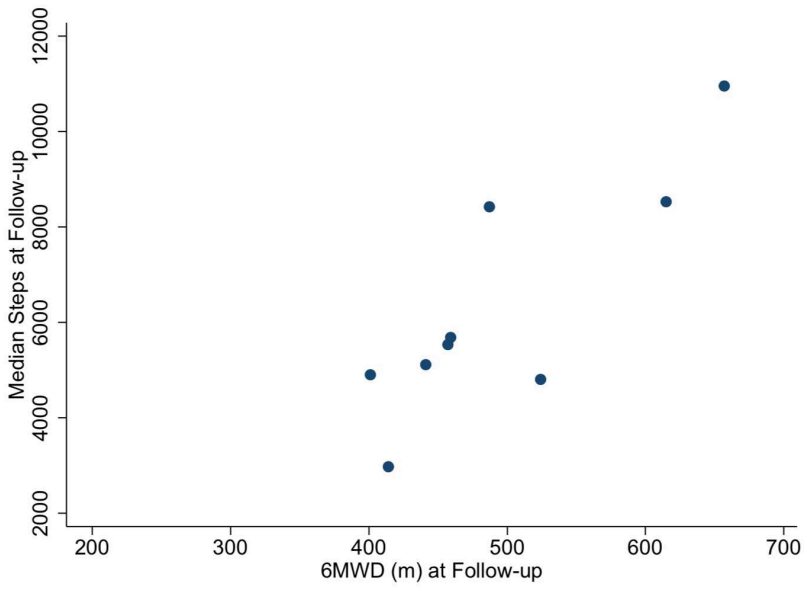

Figure 4b: 6MWD vs Fitbit Steps at Follow-up 


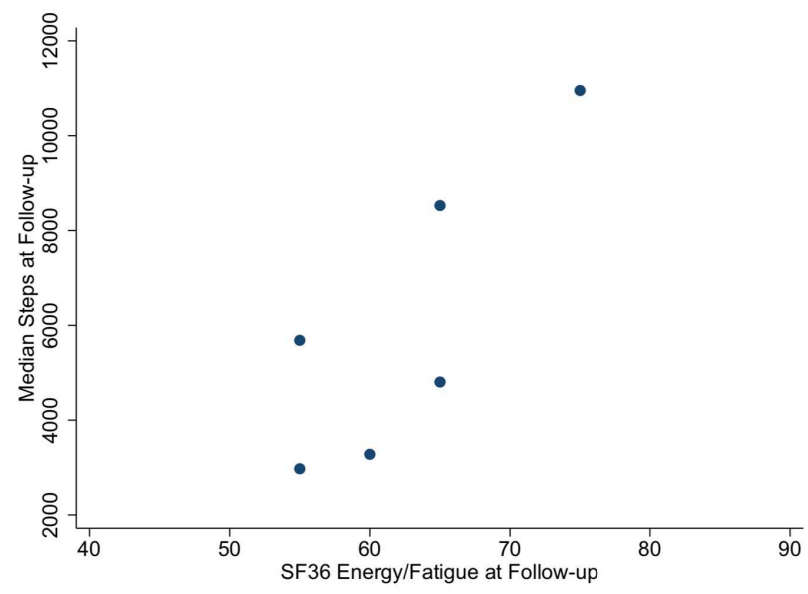

Figure 5: Fitbit Steps vs. Vitality at Follow-up 
Supplementary Table 2b: Exericse Testing Sample Size

\begin{tabular}{|c|c|c|c|c|c|c|}
\hline & $\begin{array}{l}\text { Baseline } \\
\text { overall }\end{array}$ & $\begin{array}{l}\text { Baseline } \\
\text { restricted }\end{array}$ & $\begin{array}{c}\text { Follow-up } \\
\text { overall }\end{array}$ & FU restricted & $\begin{array}{l}\text { Change } \\
\text { overall }\end{array}$ & $\begin{array}{l}\text { Change } \\
\text { restricted }\end{array}$ \\
\hline Steps (4 days) & 21 & 10 & 10 & 10 & 10 & \\
\hline 6MWD (m) & 18 & 8 & 16 & 8 & 16 & 8 \\
\hline 6MWT (steps) & 16 & 9 & & & - & - \\
\hline Borg & 17 & 8 & 15 & 8 & 15 & 8 \\
\hline $\mathrm{VO} 2 \mathrm{ml} / \mathrm{kg}$ & 20 & 9 & 14 & 9 & 14 & 9 \\
\hline VO2 \% pred. & 20 & 9 & 14 & 9 & 14 & 9 \\
\hline Watts & 17 & 6 & 11 & 6 & 11 & 6 \\
\hline Watts \% pred. & 17 & 6 & 11 & 6 & 11 & 6 \\
\hline RER & 20 & 9 & 14 & 9 & 14 & 9 \\
\hline $\mathrm{PetCO}^{2}$ & 20 & 8 & 13 & 8 & 13 & 8 \\
\hline $\mathrm{VeVCO}^{2}$ & 9 & 2 & 6 & 2 & 6 & 2 \\
\hline $\mathrm{O}^{2}$ sat rest (CPET) & 19 & 8 & 14 & 8 & 14 & 8 \\
\hline $\mathrm{O}^{2}$ sat exercise (CPET) & 6 & 1 & 2 & 1 & 2 & 1 \\
\hline
\end{tabular}




\begin{tabular}{|c|c|c|c|c|c|c|}
\hline $\mathrm{O}^{2}$ sat rest $(6 \mathrm{MWT})$ & 11 & 5 & 8 & 5 & 8 & 5 \\
\hline $\mathrm{O}^{2}$ sat exercise (6MWT) & 6 & 1 & 2 & 1 & 2 & 1 \\
\hline \multicolumn{7}{|l|}{ SF-36 } \\
\hline $\begin{array}{l}\text { Physical } \\
\text { functioning }\end{array}$ & 20 & 6 & 6 & 6 & 10 & 6 \\
\hline $\begin{array}{l}\text { Role limitations } \\
\text { due to physical } \\
\text { problems }\end{array}$ & 20 & 6 & 6 & 10 & 10 & 6 \\
\hline $\begin{array}{l}\text { Role limitations } \\
\text { due to emotional } \\
\text { problems }\end{array}$ & 19 & 6 & 6 & 10 & 10 & 6 \\
\hline Energy/fatigue & 19 & 6 & 6 & 10 & 10 & 6 \\
\hline $\begin{array}{l}\text { Emotional well- } \\
\text { being }\end{array}$ & 20 & 5 & 5 & 9 & 9 & 5 \\
\hline Social functioning & 19 & 5 & 5 & 9 & 9 & 5 \\
\hline Pain & 20 & 6 & 6 & 10 & 10 & 6 \\
\hline General health & 20 & 6 & 6 & 10 & 10 & 5 \\
\hline Health change & 20 & 5 & 5 & 9 & 9 & 5 \\
\hline
\end{tabular}

